\title{
Design and Implementation of Wireless Monitoring System for Single-resided Elder
}

\author{
Wei WANG \\ Department of Embedded System Engineering, \\ Dalian Neusoft University of Information \\ Dalian, China \\ e-mail: wangwei_q@neusoft.edu.cn \\ Yonggang MA \\ School of Science \\ Dalian Ocean University \\ Dalian, China \\ e-mail: mayonggang2006@hotmail.com \\ Yiming LI \\ Department of Embedded System Engineering, \\ Dalian Neusoft University of Information \\ Dalian, China
}

\begin{abstract}
The ageing population increases significantly in these years. How to care for the elderly people living alone becomes an important problem. This design is a wireless monitoring system for elderly people living alone based on ZigBee and LabVIEW because ZigBee is a low power and low cost wireless networking technology and LabVIEW is a graphical programming platform. This system performs monitoring for daily activities of the elderly by setting a variety of different parameter sensors in the room and the monitoring data are sent to the monitoring center wirelessly. Then the monitoring personnel can monitor the daily activities of the elderly. When there are some abnormal situations occurring in the elderly, the system can immediately sound the alarm and inform their families. This design can make the elderly living alone get proper care. Therefor this system is an economic and effective monitoring system for the elderly people.
\end{abstract}

Keywords-LabVIEW; ZigBee; Wireless monitoring;The upper computer; The low computer

\section{INTRODUCTION}

The ageing trends become more and more obvious especially in big cities. How to care for the older people has become increasingly concerned in the word [1,2]. So it is necessary to design and implement a monitoring system for the elderly people to care for their lives [2,3]. There exist many different monitoring systems for the elderly people [4-9]. Along with the rapid development of new technologies and hardware products, we design and implement a new economic and effective monitoring system. This design mainly uses ZigBee wireless networking technology and LabVIEW to accomplish the nursing function.

\author{
Song YANG \\ Department of Embedded System Engineering, \\ Dalian Neusoft University of Information \\ Dalian, China
}

\author{
Nan DING \\ School of Computer Science and Technology \\ Dalian University of Technology \\ Dalian, China
}

\section{THE OVERALL SYSTEM DESIGN}

In order to save the cost, the design chooses ZigBee as the wireless networking to accomplish the wireless data transmission instead of the GPRS, because the GPRS needs to charge a fee. The ZigBee also has the characters of short distance, low complexity, low power consumption and low data rate [10-12]. Because the ZigBee is subject to some restrictions on the transmission distance, this design is mainly fit for the higher-density residential areas and not far away from each other. The system mainly consists of two parts: the upper computer and the lower computer. The upper computer is a PC that is mainly used to send the commands to the lower computer and display the monitoring results in Community Care Management Center. The lower computer is mainly composed of a variety of sensor acquisition modules, data acquisition modules, routers and coordinators. The sensor acquisition modules collect the indicator, status and other data of the older people's lives. The collected data such as room temperature, fume and pulse are transferred to the collectors via RS-485 bus. Then the collectors complete the data exchange via the routers and coordinators. The coordinators upload information to the data management platform of the upper computer.

For those people in need of supervision in a unit of a building, the system implements data set copy through the RS-485 bus. This constitutes a ZigBee network node and this network node communicates with the upper routers. Then the data are uploaded to a coordination device through the ZigBee network. The collector nodes are usually placed in a residential roof because this placement can reduce the environmental interference to the devices. The data are transmitted among the network nodes 
wirelessly based on ZigBee protocol. The overall system block diagram is shown in Fig.1.

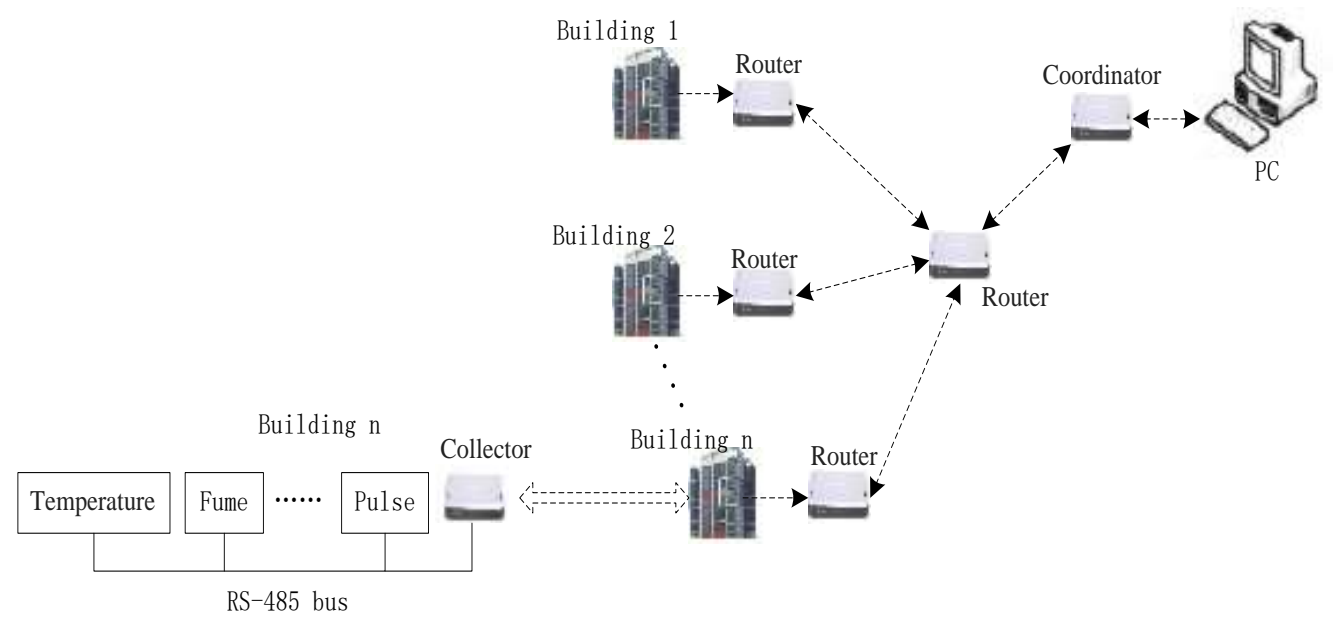

Figure 1. The Overall Block Diagram of the System

\section{THE LOWER COMPUTER HARDWARE DESIGN}

We select the low-power System on Chip CC2530 RF chip that is made by TI Company as the core microprocessor of ZigBee radio frequency (RF) module. The CC2530 has lower power consumption compared with the first-generation chip CC2430. Moreover the CC2530 has maximum communication distance of $400 \mathrm{~m}$ and up to $256 \mathrm{~KB}$ of flash memory. So it can support wide applications. Its powerful address recognition and packet processing engine can be a good match for RF front end and the package is much smaller.

\section{A. Acquisition and Router Hardware Design}

The main tasks of acquisition are to receive the host computer instructions and read data from each sensor module of the underlying connection in accordance with the requirements of the instructions. And then the acquisition sends the data to the host computer through the wireless network. The primary functions of the router are to extend the transmission range of the wireless sensor network and forward the communication command and data information between the coordinator and the acquisition. The collector connects the sensing modules via a standard RS-485 serial interface. According to the RS-485 communication protocol, the messages are sent to the collector and each sensor module returns the logger to the collector by the RS-485 interface. Then the collector passes these data acquisition packages to the coordinator through the wireless network. The coordinator uploads the data packets to the host computer. The acquisition and router hardwares are mainly made up of CC2530 wireless module, RS-485 interface circuit, sensor module and power supply circuit. The collector and router structure diagram is shown in Fig .2.

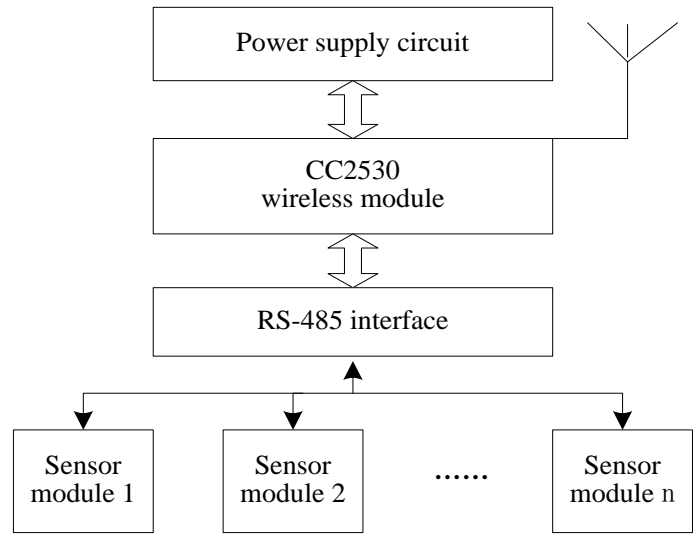

Figure 2. The Collector and Router Schematic Diagram

\section{B. The Coordinator Hardware Design}

The coordinator hardware architecture is shown in Fig .3. The coordinator is mainly responsible for starting, configuring and coordinating the entire ZigBee network. The coordinator is also responsible for sending the command and data information from the data management center to any ZigBee network devices. Meanwhile the coordinator is responsible for receiving the monitoring data returned by any devices in ZigBee network and uploading the data to the PC in Community Management Center. The coordinator is the core unit of the monitoring system. It serves as a bridge across the communication system and determines the performance of the entire system. 


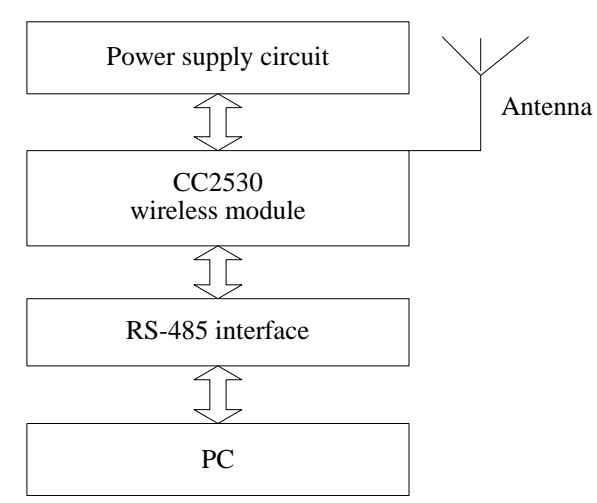

Figure 3. The Coordinator Schematic Diagram

\section{THE LOWER COMPUTER SOFTWARE DESIGN}

The system uses the software to monitor and analyze the activities of the elderly. When the system receives the abnormal information or does not receive the monitoring data, the system determines an emergency occurring and sounds an alarm. Meanwhile the system will notify the center management personnel immediately to check the home. If some problems occur with the elder, the monitoring personnel will take some measures to deal with the emergency and inform the families of the elderly people right now.

\section{A. The Acquisition Software Design}

The acquisition program flowchart is shown in Fig .4. After the acquisition receives the command from the coordinator, the collector will compare the user addresses with the addresses that the collector is responsible for managing. If the address is within the scope of this module that is responsible for, the collector will send the appropriate user data message to the coordinator.

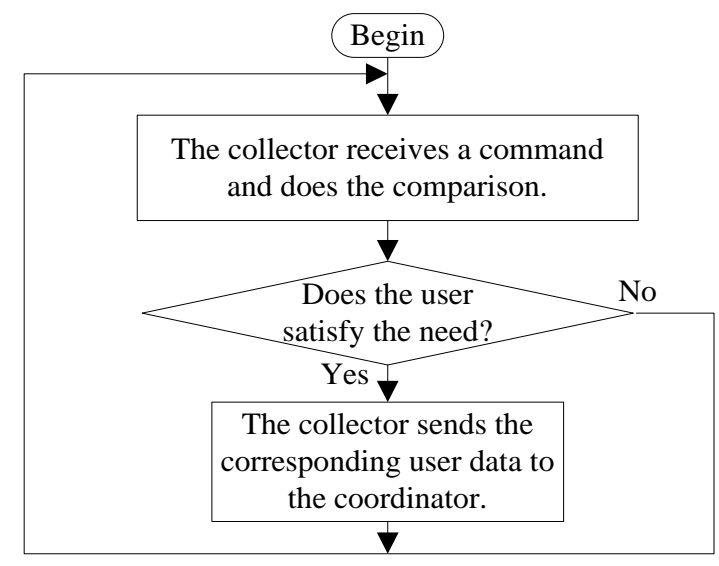

Figure 4. The Acquisition Program Flowchart

\section{B. The Coordinator Software Design}

The coordinator software design flowchart is shown in Fig .5. The coordinator firstly initializes the serial port including the baud rate setting and interruption, and then establishes a network in a way of broadcast. And then the coordinator monitors the serial port. When the serial port events happen, the coordinator will send the serial port command and receive the corresponding collected data. After that, the coordinator will determine whether there is a new acquisition registration. If a new acquisition registers, the coordinator will process it and it becomes a new network node.

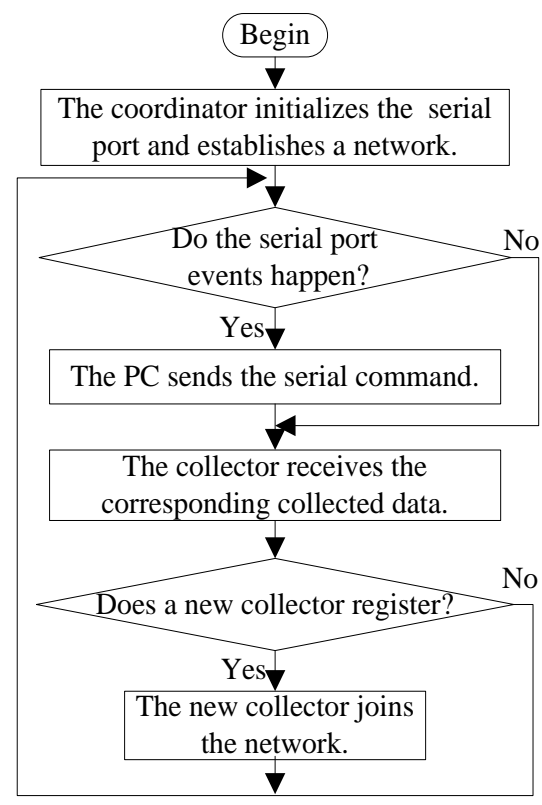

Figure 5. The Coordinator Program Flowchart

\section{THE PC SOFTWARE DESIGN}

The PC serial communication procedures are programmed in LabVIEW language that is a program development environment developed by National Instruments (NI) Company. LabVIEW is a graphical editor $\mathrm{G}$ programming language and the program is produced in block diagram form, which helps engineers scale from design to test and from small to large systems.

We can select these users who need to be queried from the drop-down list. Then the program will send different commands to the terminals through the serial ports. The returned data from the terminals can be displayed by a graphical interface. The PC program is mainly responsible for controlling the lower computer and reading and displaying the information. The major functions used in this design are: (1) VISA serial interrupt; (2) VISA serial number of bytes; (3) VISA read; (4) VISA close; (5) VISA serial port configuration. The core of the program flowchart is shown in Fig .6.

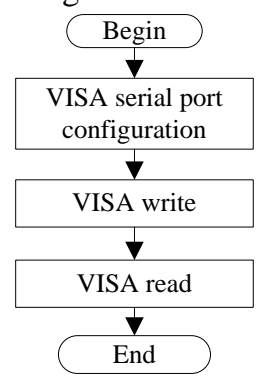

Figure 6. The Core Part of the Program Flowchart 
The data display section using LabVIEW is shown below. This section can select the data to be displayed by string interception control. Through the control display and then by the string/numeric conversion control, the data are converted to numerical value. So the information can be displayed through the instrument and waveform diagram controls. This display is more intuitive and easy to observe. The data display diagram is shown in Fig .7.

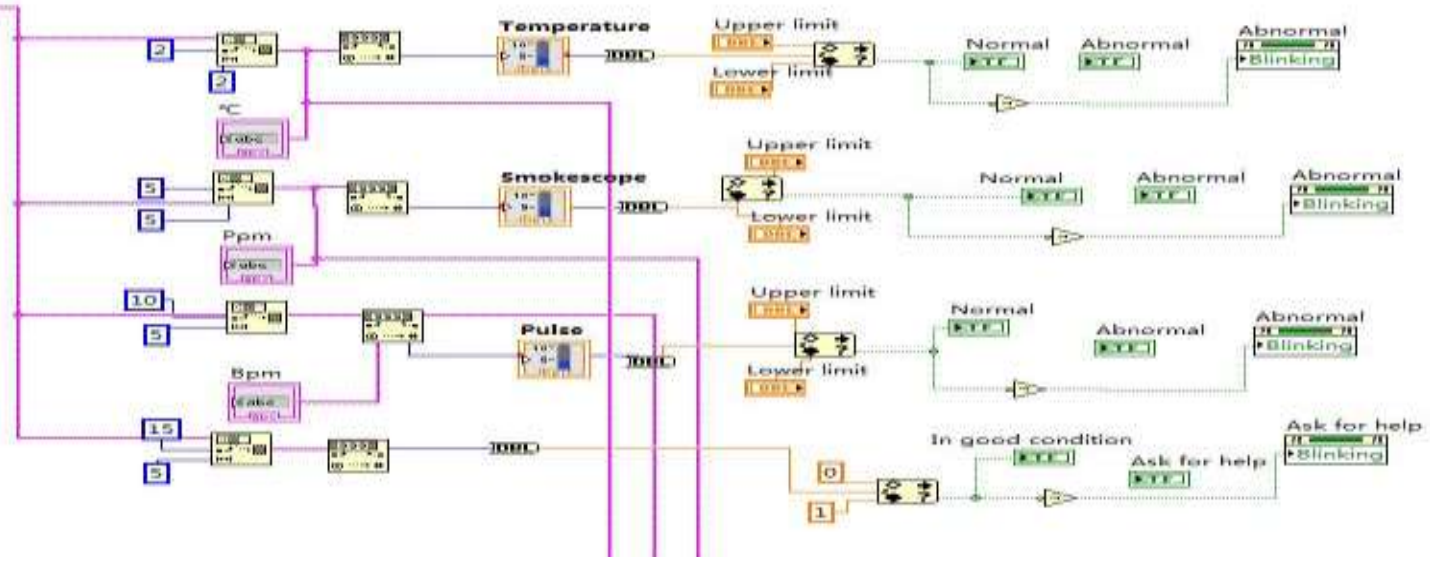

Figure 7. The Data Display Diagram

\section{SIMULATION TESTS}

To test the actual effect of the collection nodes, we have performed some simulation tests in a lab environment. The analog network consists of a coordinator, a router, two collectors and three sensor modules. These devices measure the indoor temperature, smoke and pulse information separately, and connect to the RS-485 bus system board. The single PC connects with the coordinator via RS-232 communication interface. We can query the monitoring data such as Building 1, Unit 1, Room 101 by setting the PC software of LabVIEW. The query result is shown in Fig .8.

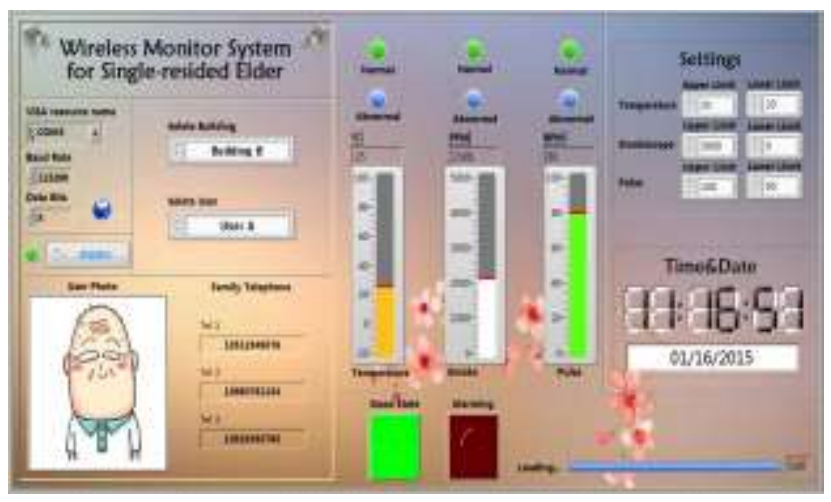

Figure 8. The PC Query Result

When we select the different unit and the house number, the program will send different commands and obtain different user data through the serial ports. The PC can also import the users' photos, so the system can display the pictures when we make some choices. This display is more intuitive and the system also has the function of voice broadcast. Then the system can broadcast the query information and display the system time.

\section{CONCLUSIONS}

The above results show that we complete the design and implementation of the monitoring system for the elderly living alone successfully. The system has several advantages such as the subsequent user cost savings, the secondary development and maintenance convenience. But there are also some shortcomings in practical applications such as the transmission distance is only a few ten meters and the communication distance is short. If we want to extend the communication range, we can add the power amplifier in the acquisition side. On the other hand, we can combine the coordinator and collector with a repeater. Then we can achieve more distance monitoring. If we want to complete multiple users simultaneous real-time monitoring and display in 24 hours, the system still need to be further optimized.

\section{ACKNOWLEDGMENTS}

This work is partially supported by the National Natural Science Foundation of China (Grant No.61471084) and the Foundation for the Excellent Doctoral of Dalian Ocean University (Grant No.HDYJ201317) and the Scientific Research Foundation of the Education Department of Liaoning Province, China (Grant No.L2014279).

\section{REFERENCES}

[1] United Nations, Department of Economic and Social Affairs, Population Division (2013). World Population Ageing 2013. ST/ESA/SER.A/348.

[2] Nadia Zouba, François Bremond, Monique Thonnat, Alain Anfosso, Éric Pascual, Patrick Mallea, Véronique Mailland, and Olivier Guerin, Assessing computer systems for monitoring elderly people living at home, Proceedings of the 19th IAGG World Congress of Gerontology and Geriatrics,: IAGG, 2009.

[3] Moshtaghi, M., Zukerman, I., Russell, R. A., Albrecht, D. Towards detecting inactivity using an in-home monitoring system. In IcIEA 2013- Proceedings of the IEEE Conference on Industrial 
Electronics and Applications, Melbourne, Australia, pp. 935-940, 2013.

[4] A. Tablado, A. Illarramendi, J. Bermúdez, A. Goñi, Intelligent Monitoring of Elderly People, Proc of the 4th Annual IEEE Conf on Information Technology Applications in Biomedicine, UK, 2003.

[5] Mann WC, Belchoir P, Tomita MR, et al, Older adults' perception and use of PDAs, home automation system, and home health monitoring system. Topics in Geriatric Rehabilitation, 2007, 23(1):35-46.

[6] Tang Mingxia. Design of the Wireless Monitor System for Singleresided Elder [D].Harbin University of Science and Technology, 2007.

[7] Larizza,M.Zukerman,I.,Bohnert,F.,Busija,L., Bentley, S., Russell,R., Rees,G., In-home monitoring of older adults with vision impairment: exploring patients', caregivers' and professionals' views, Journal of the American Medical Informatics Association, 2013, 21(1), 56-63.
[8] Kasteren,T.L., Englebienne,G. Krose, B.J, An activity monitoring system for elderly care using generative and discriminative models, Personal Ubiquitous Computing, 2010, 14(6), 489-498.

[9] Shigeru Ohta, Hiroshi Nakamoto, Yoshimitsu Shinagawa,Tomohiro Tanikawa, A Health Monitoring System for Elderly People Living Alone, Journal of Telemed Telecare, 2002, $8,151-156$

[10] Wei Jiang; Zhaoli Zhang, Networked wireless meter reading system based on ZigBee technology, Chinese Control and Decision Conference (CCDC 2008), 3455- 3460, 2008.

[11] Primicanta, A.H., Nayan, M.Y., Awan, M., ZigBee-GSM based Automatic Meter Reading system, 2010 International Conference on Intelligent and Advanced Systems (ICIAS), Kuala Lumpur, Malaysia, 1 1-5, 2010.

[12] Wang Yinfang, Ma Shiwei. Research and Design of Wireless Meter Reading System Based on ZigBee [J]. Measurement and Control Technology, 2013, 32(1), 14-18. 